\title{
Decrease in Cellular Nanovesicles Concentration in Blood of Athletes More Than I5 Hours After Marathon
}

This article was published in the following Dove Press journal: International Journal of Nanomedicine

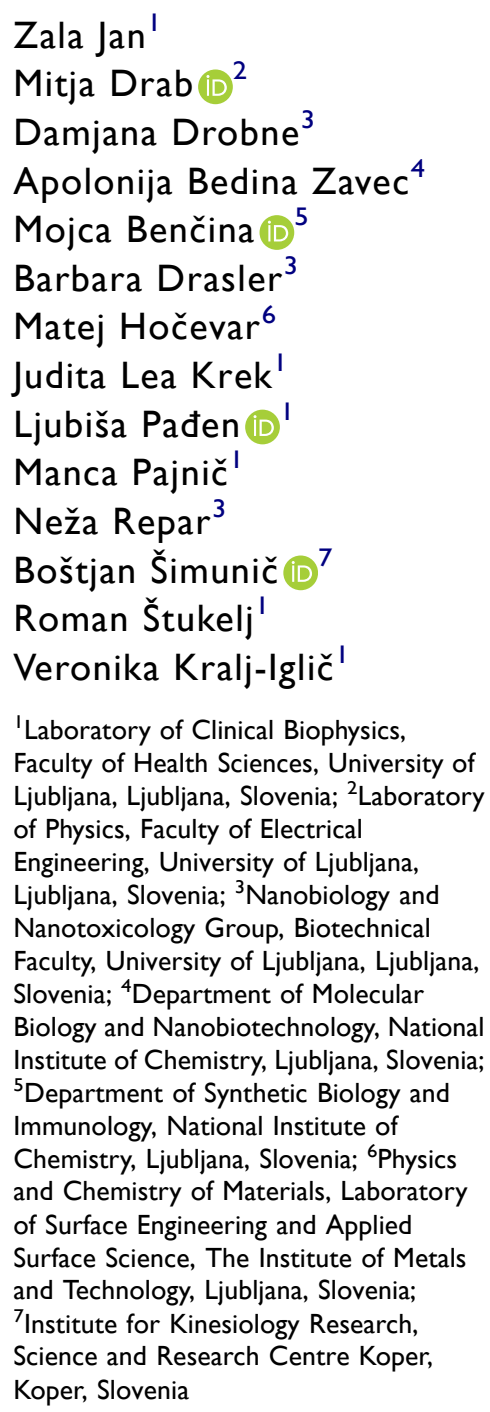

Correspondence: Veronika Kralj-Iglič Laboratory of Clinical Biophysics, Faculty of Health Sciences, University of Ljubljana, Ljubljana, Slovenia

Email kraljiglic@gmail.com
Introduction: Cellular nanovesicles (CNVs), that are shed from cells, have been recognized as promising indicators of health status. We analyzed the effect of long-distance running on concentration of CNVs, along with some standard blood parameters, in 27 athletes two days before and $>15$ hours after physical effort.

Methods: CNVs were isolated by repetitive centrifugation and washing of samples, and assessed by flow cytometry. Cholinesterase (ChE) and glutathione S-transferase (GST) activity were measured spectrophotometrically. Interleukin 6 (IL-6) and tumor necrosis factor- $\alpha(\mathrm{TNF}-\alpha)$ concentrations were measured using enzyme-linked immunosorbent assay (ELISA). C-reactive protein (CRP) was measured with immunoturbidimetric determination and lipidogram parameters were measured by enzymatic colorimetric assay. Flow cytometry was used for blood cell count and mean platelet volume (MPV) measurement.

Results: More than 15 hours after physical effort a decrease was found in CNVs' concentration in isolates from blood ( $46 \%$; $<<0.05)$, in ChE activity in whole blood (47\%; $<<0.001)$, in plasma (34\%; $<<0.01)$, and in erythrocyte suspension (54\%; $<<0.001)$, as well as in GST activity in erythrocyte suspension $(16 \% ; \mathrm{p}<0.01)$ and in IL-6 concentration in plasma $(63 \%$; $\mathrm{p}<0.05)$. We found no change in GST activity in plasma and in TNF- $\alpha$ concentration in plasma. Correlations $(>0.8 ; \mathrm{p}<0.001)$ between $\mathrm{CNVs}$ ' concentration and $\mathrm{ChE}$ activity, and GST activity, respectively, in erythrocyte suspension were found.

Conclusion: We found that $>15$ hours post-physical effort, CNVs' concentration was below the initial value, concomitant with other measured parameters: ChE and GST activity as well as IL-6 concentration, indicating a favorable effect of physical effort on health status. CNVs' concentration and ChE activity in isolates from peripheral blood proved to have potential as indicators of the response of the human body to inflammation after physical effort. Physical activity should be considered as an important factor in preparation of subjects for blood sampling in procedures focusing on CNV-containing diagnostic and therapeutic compounds. Keywords: membrane vesiculation, physical effort, blood samples, inflammation process, cellular nanovesicles, marathon

\section{Introduction}

Regular physical effort offers protection against all-cause mortality, elevates the metabolic rate and increases oxygen consumption by the entire body, particularly by locomotive muscles, which evokes acute stress response with consequent triggering of instant changes in physiological parameters and in homeostatic processes in the human body. ${ }^{1}$ Due to stress and immune response mechanisms during exercise, 
several tissues release molecules into the blood stream, with the aim to mediate stress-related effects throughout the whole body. ${ }^{2}$ There is evidence that acute physical effort involves changes in nerve conduction signals, ${ }^{3}$ oxidative stress ${ }^{4}$ and inflammatory processes. ${ }^{5,6}$ Regular physical effort, especially aerobic exercise, reduces morbidity and mortality and results in adaptation of endocrine, cardiovascular and immune systems to oxidative stress and inflammation caused by physical effort. ${ }^{7}$

Under the term "Cellular Nanovesicles" (CNVs) we mean sub-micron sized membrane-enclosed fragments that can be found in isolates (in this work, we consider isolates from blood). A related term "Extracellular vesicles (EVs)" is defined as a "generic term for particles naturally released from the cell that are delimited by a lipid bilayer and cannot replicate". 8 The difference between the two terms is in the definition of the system and in the suggested origin of the particles. Namely, "CNVs" that pertain to isolates also include particles that are potentially formed during the processing of samples. CNVs more realistically interpret the particles in the isolates, since the samples are subjected to procedures and conditions that likely cause cell fragmentation and fragment remodeling. "EVs" pertain to in vivo systems, which can however not be directly observed without affecting the system. EVs can be regarded as a subset of CNVs (ie, EVs are those CNVs that are shed by the cells in the surroundings and their identity remains unchanged during the harvesting). As there is to our knowledge no method that could prove the mechanism of formation of particles in the isolates solely by analyzing the isolates, we think that "CNVs" is an appropriate term when addressing the isolates while "EVs" is an appropriate term when addressing the suggested in vivo mechanisms. CNVs were hitherto isolated from different body fluids and visualized by electron microscopy. ${ }^{34}$

It was suggested that EVs are formed in the final stage of membrane budding ${ }^{10}$ by pinching off of the buds from the cell membranes and that they present an intercellular communication system within the body. ${ }^{11,12}$ EVs discharged into the circulation could assist with disposal of cellular waste products generated under stress conditions and help the cells to preserve homeostasis. ${ }^{13} \mathrm{EV}$ formation by cells is considered a physiological process ${ }^{14}$ that can be accelerated by oxidative stress ${ }^{15}$ and by inflammatory process. ${ }^{6}$ EVs may have an important role in microvascular, ${ }^{16}$ skeletal muscle and systemic ${ }^{14}$ adaptation to physical effort.
Previous observations indicated an increased concentration of CNVs in body fluid isolates during physical effort, immediately after physical effort and also after up to four hours of rest. ${ }^{2,9-13}$ An increased concentration of CNVs in isolates may be connected to the release of heat shock proteins, which provoke acute stress response of the body during exercise. ${ }^{13}$ Most of CNVs found in blood isolates during physical effort contain receptors characteristic of endothelial cells and blood cells, in particular, activated platelets. ${ }^{17}$ Increase in concentration of plateletorigin-CNVs has been previously observed after acute aerobic exercise. ${ }^{9,18}$ The mechanisms leading to this effect are not fully understood.

Physical effort also enhances other physiological staterelated parameters that reflect inflammation and stress response of the human body, ${ }^{19}$ such as cholinesterase $(\mathrm{ChE})^{3}$ and glutathione S-transferase (GST) activity ${ }^{4}$ and concentrations of cytokines ${ }^{6,20}$ and C-reactive protein $(\mathrm{CRP})^{5}$ in blood samples. It was found that two types of $\mathrm{ChE}$, acetylcholinesterase (AChE) and butyrylcholinesterase $(\mathrm{BuChE})$, may enhance inflammation with inactivation of neurotransmitter acetylcholine (ACh) that is believed to modulate immune and anti-inflammatory response via the cholinergic system; a key factor in the anti-inflammatory cholinergic pathway is supposed to be oxidative stress. ${ }^{21}$ Some researchers found that $\mathrm{ChE}$ activity in blood samples is influenced by physical effort, ${ }^{22}$ yet others found no effect in athletes. ${ }^{3}$ It was suggested that decrease in erythrocyte suspension AChE activity may reflect an increased response of the human body to oxidative stress which was associated with increased metabolism due to prolonged physical exercise. ${ }^{23}$ GSTs are a family of antioxidant enzymes that act in antioxidant defense, help in decreasing reactive oxygen species and in protecting cells from oxidative stress. ${ }^{24}$ Erythrocyte suspension GST activity has been proposed as a potential marker for oxidative stress. ${ }^{25}$ However, evidence on GST in connection with physical effort is limited. On the contrary, there is much evidence regarding the inflammatory processes in physical effort. CRP is an acute phase protein synthetized primarily in the liver, but also in kidneys, neurons, and bowel; its concentration in plasma is connected to inflammatory processes and tissue damage. ${ }^{26}$ Interleukin-6 (IL-6) is a myokine, produced during aerobic and resistance physical effort when muscles are under contraction; it acts as an inflammatory mediator. ${ }^{5}$ IL-6 is involved in mediating the health-beneficial effects of exercise and protects the human body against chronic diseases such as diabetes and cardiovascular diseases, both 
associated with low-grade inflammation. ${ }^{20}$ Tumor necrosis factor- $\alpha$ (TNF- $\alpha$ ) was originally described as a circulating factor that can cause necrosis of tumors but has since been identified as a key regulator of the inflammatory response. ${ }^{27}$ Delayed release of cytokines following eccentric exercise was found to be related to the repair of muscle damage and the production of cytokines turned out to be greater with endurance than with resistance exercise. ${ }^{28}$ Systemic or acute-phase inflammation can be detected by monitoring CRP production, stimulated by cytokines IL- 6 and TNF- $\alpha$, indicators of local inflammatory response. ${ }^{20}$ It was found that after strenuous exercise, transient short-term increase in plasma CRP occurs; on the other hand, chronic physical effort provokes multiple mechanisms that reduce resting CRP circulating concentrations. ${ }^{5}$

It is acknowledged that isolates are sensitive to processing procedures (blood sampling, handling the samples, external parameters such as temperature, and time course of experiments) and to the donor status. ${ }^{29}$ Physical effort is expected to influence the release of CNVs. ${ }^{2,9-13}$ Overnight fasting before blood collection in the morning, avoidance of strenuous physical effort the day before sampling and advice against using anti-inflammatory products are presently the guidance given to the blood donors. ${ }^{29}$ However, the underlying mechanisms are not completely understood.

Lipidogram parameters and blood cell parameters are of interest due to possible interference with concentration of CNVs isolated from peripheral blood and possible risk for thromboembolism. It has been previously shown that the concentration of CNVs in peripheral blood correlates with total blood cholesterol and the prandial state of the individual. ${ }^{30}$ As reported by Brahmer et al (2019), ${ }^{2}$ blood cell count of erythrocyte suspensions, platelets and leukocytes as well as concentration of lipoproteins, including high density lipoproteins (HDL), low density lipoproteins (LDH) and triglycerides (TGL) are also connected with the concentration of CNVs in isolates from blood. Mean platelet volume (MPV) is considered to be a marker of platelet activation, ${ }^{31}$ which is also connected to vesiculation of platelets. $^{32}$

In the study presented here we studied possible change of CNV concentration in isolates from peripheral blood samples and concomitant change of some physiological state-related blood parameters that reflect response to inflammation and stress (ChE and GST activity, IL-6 and TNF- $\alpha$ concentrations) as well as some standard blood parameters. We compared these quantities two days before and $>15$ hours after long-distance running. We discussed the applicability of CNVs concentration and ChE activity as indicators of the response of the human body to inflammation after physical effort. Understanding these mechanisms and using the knowledge in planning of physical activities will decrease risk for adverse effects of physical effort and improve physical performance in various fields, including recreational and competitive sport. Also, understanding the effects of physical activity on the assessment of CNVs in isolates will improve the repeatability and accuracy of CNV-based diagnostic and therapeutic methods by instructing the blood donors how to prepare for blood sampling.

\section{Materials and Methods \\ Participants}

We included 27 volunteers (15 females, 12 males) in the study, aged from 24 to 62 years, with a mean age of 36 (9.6) years who participated in 20th Ljubljana Marathon. All participants gave written consent and the study was conducted according to the rules of The Declaration of Helsinki and has been approved by Republic of Slovenia National Medical Ethics Committee, (approval number: 82/07/14). All participants were informed about the purpose of the study. All participants were declared healthy, with one participant being a type 1 diabetic. All participants ran a distance of at least $10 \mathrm{~km}$ (14 of them ran $21 \mathrm{~km}, 4$ ran a full $42 \mathrm{~km}$ marathon, one of whom stopped at $34 \mathrm{~km})$.

\section{Blood Sampling}

Blood was collected twice: first, two days before the marathon, when all of the participants were pre-prandial for at least 8 hours (mean 11.3 hours), and second $>15$ and $<22$ hours after the marathon, when the participants were pre-prandial for at least 10 hours (mean 11.1 hours). Whole blood samples were collected by medial cubital vein puncture using a 21-gauge needle (length $70 \mathrm{~mm}$, inner radius $0.4 \mathrm{~mm}$, Microlance ${ }^{\circledR}$, Becton Dickinson, Franklin Lakes, NJ, USA) and stored in four $2.7 \mathrm{~mL}$ vacutubes, thermostated at $37^{\circ} \mathrm{C}$ before the blood sampling. Vacutubes for CNVs, GST, ChE, IL- 6 and TNF- $\alpha$ assessment contained $270 \mu \mathrm{L}$ trisodium citrate $(0.109 \mathrm{~mol} /$ L) as an anticoagulant. For further hemogram analysis Ethylenediaminetetraacetic acid (EDTA) was used as an anticoagulant and for biochemical analysis, no anticoagulant was added. 


\section{Preparation of Samples}

After the blood collection, vacutubes were gently mixed by inverting. The samples were processed immediately when all the sampling within the given group was completed. No sample waited more than half an hour. While waiting, the samples were kept in thermoblocks at $37^{\circ} \mathrm{C}$. From vacutube with trisodium citrate, $100 \mu \mathrm{L}$ of the blood sample was dispensed into Eppendorf tube for whole blood ChE activity analysis. Remainder of the sample and sample from other vacutube with trisodium citrate were combined in a $15 \mathrm{~mL}$ Falcon tube (to yield app. $8 \mathrm{~mL}$ of whole blood) for each donor. The samples were then centrifuged for $20 \mathrm{~min}$ at $1550 \times \mathrm{g}$ and $37^{\circ} \mathrm{C}$. After the centrifugation, the pellet contained a great majority of erythrocytes and the supernatant consisted of platelet poor plasma, with a fuzzy layer of white cells between them. Pellet was used for erythrocyte suspension $\mathrm{ChE}$ and GST activity analysis. The supernatant was divided in the following manner: top $250 \mu \mathrm{L}$ for CNV analysis, $250 \mu \mathrm{L}$ for GST and ChE activity analysis, and $100 \mu \mathrm{L}$ for inflammation factor analysis.

\section{Isolation from Blood Plasma and Assessment of CNVs}

$\mathrm{CNV}$ isolation from platelet poor plasma was prepared according to a protocol that was previously elaborated as regards blood sampling, ${ }^{33}$ visualization of the isolate ${ }^{34}$ and variation of temperature. ${ }^{34}$ It was found that this method yields in the isolates the particles that comply with theoretically obtained shapes corresponding to the minimum of the membrane free energy. ${ }^{34}$ Also, it was found that a large pool of the particles was formed in the process of isolation, however, the numbers of CNVs in the isolates reflect the properties of blood cells and plasma and are therefore clinically relevant. ${ }^{35}$ For details regarding the identity of the particles in the isolates obtained by the method also used in this work please see references 33 and 35. To isolate CNVs, plasma was pipetted into $1.5 \mathrm{~mL}$ Eppendorf tubes and centrifuged at $17,570 \times g$ and $37^{\circ} \mathrm{C}$ for 5 minutes. The top $225 \mu \mathrm{L}$ of supernatant was removed and replenished with PBS-citrate buffer. After the second centrifugation $210 \mu \mathrm{L}$ of supernatant was removed, and the tube was replenished with $60 \mu \mathrm{L}$ PBScitrate buffer to yield the isolate.

The isolate was assessed by flow cytometry as described in detail in references 33 and 35 . The sample was vortexed at $1200 \times g$ for 10 seconds, then $100 \mu \mathrm{L}$ of the sample was pipetted into the measuring chamber of a MACS Quant flow cytometer (Miltenyi Biotec GmbH, Bergisch-G Ladbach, Germany) with $405 \mathrm{~nm}, 488 \mathrm{~nm}$, and $640 \mathrm{~nm}$ air-cooled lasers. Flow cytometer was calibrated using the MACSQuant Calibration Beads, $2 \mu \mathrm{m}$ and $3 \mu \mathrm{m}$ in size. The presence of CNVs and residual cells was determined within the forward scatter parameters and side scatter parameters (FFC and SSC axes on the scatterplot, respectively). ${ }^{36}$ To adjust the channels and set the gate, $460 \mathrm{~nm}$ beads were used as a reference to the CNVs in the plasma sample. For all channel settings, the hyper log (hlog) settings were selected. The threshold or trigger on side scatter parameters (SSC) was set to 4 and secondary trigger was shut off. Events in buffer (PBS citrate) were measured and excluded as the background. The result of the measurement corresponding to CNVs was the total number of detectable particles in the plasma sample per $\mu \mathrm{L}$. A typical scatter plot before and after marathon (as obtained by this method) is shown in Figure 1 (Panels A and B, respectively). At least 10,000 events were recorded for each sample. MACSQuantifyT v. 2.4 software (Miltenyi Biotec $\mathrm{GmbH}$, Bergisch-Gladbach, Germany), was used for data acquisition and results analysis. The results were expressed in number of $\mathrm{CNVs} / \mathrm{mL}$ of plasma - CNVs concentration.

Isolates of CNVs were visualized by scanning electron microscopy (SEM). ${ }^{33,35}$ Samples were fixed, goldsputtered and observed by the JEOL JSM-6500F scanning electron microscope (JEOL, Japan). A typical image of the isolate is shown in Figure 2. The shapes of the particles have characteristics of membrane-enclosed particles without internal structure - membrane-enclosed vesicles. ${ }^{35}$ Immunolabeling showed that the CNVs obtained by this method carry receptors that are found in membranes of endothelial cells, platelets, and erythrocytes. ${ }^{33}$

\section{Glutathione S-Transferases (GST) Activity Assessment}

GST activity in red blood cells and plasma was measured using the method previously described by Mannervik. ${ }^{37}$ Red blood cells were diluted 1:300 in PBS $(\mathrm{pH}=6,5)$ with $0.1 \%$ Triton X-100 and plasma samples were used undiluted. $70 \mu \mathrm{L}$ samples were transferred to a 96-well microtiter plate and $180 \mu \mathrm{L}$ of reaction mixture was added. The reaction mixture consisted of $1 \mathrm{mM}$ ethylenediaminetetraacetic acid (EDTA) in PBS (pH 6.5), $1 \mathrm{mM}$ glutathione (GSH), and $1 \mathrm{mM}$ 1-chloro-2,4-dinitrobenzene (CDNB). Formation of the CDNB-GSH conjugate was measured spectrophotometrically at room temperature by monitoring 

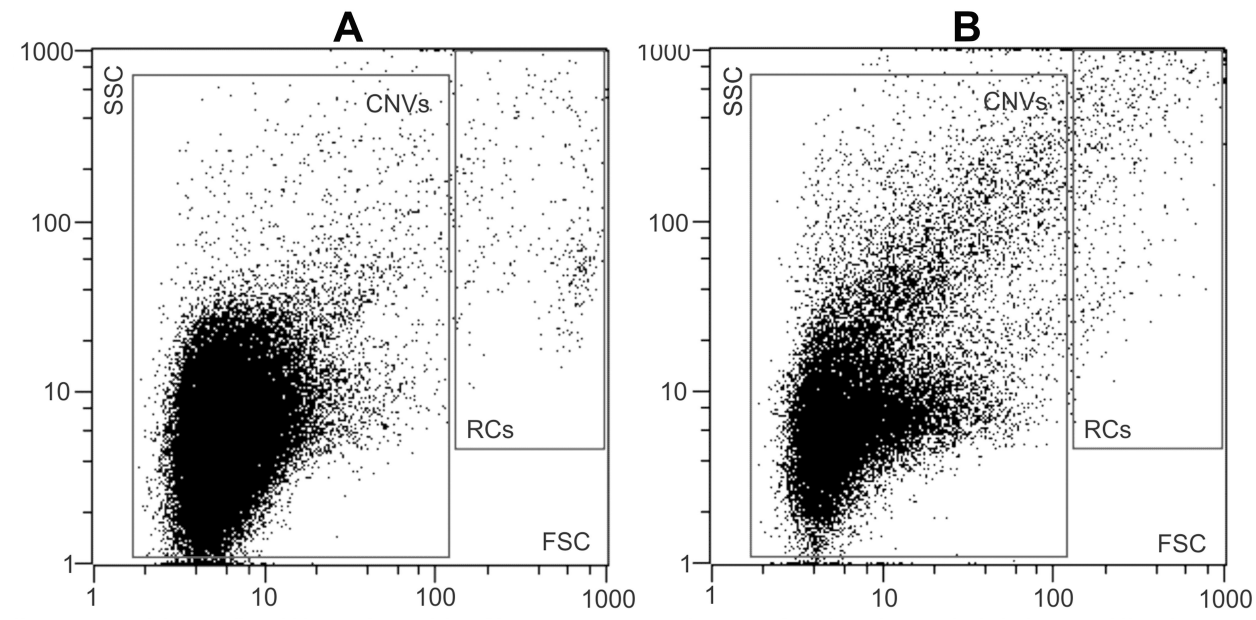

Figure I Gating strategy for the analysis of CNVs by FCM. 2D-plot of typical CNV isolates, prepared from blood plasma of a donor before (A) and after (B) the marathon. Gating of the CNVs and residual cells (RCs) was as indicated. The total number of events in the CNVs gate were taken.

Abbreviations: CNVs, cellular nano vesicles; RCs, residual cells; FCM, Flow Cytometry; FSC, forward scatter; SSC, side scatter.

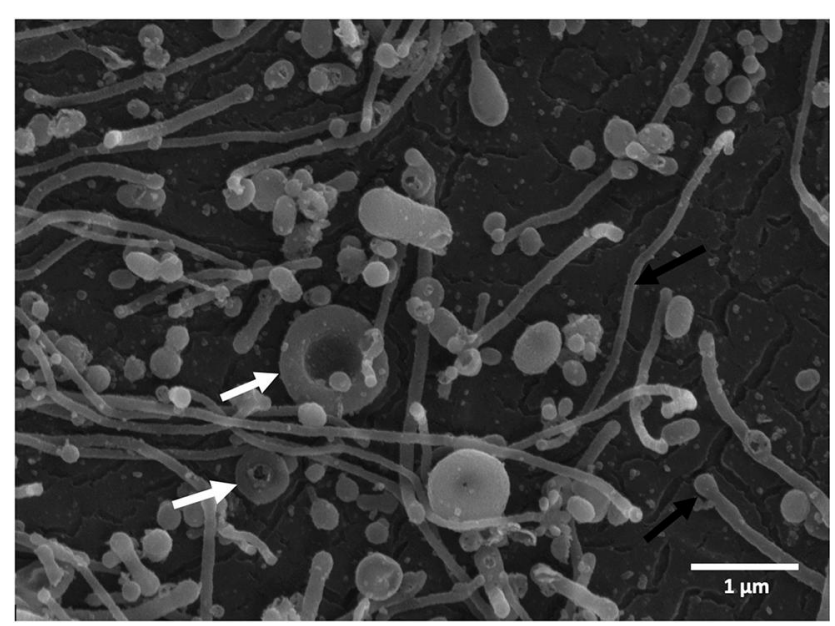

Figure 2 Scanning electron micrograph of a typical isolate of CNVs from the blood plasma sample with characteristic shapes of particles without internal structures including spheres, tubules (black arrows), and tori (white arrows). No residual cells are observed in the figure.

the change in absorbance at $340 \mathrm{~nm}$ every minute over a period of 12 minutes (BioTek, Cytation 3, Bad Friedrichshall, Germany). Each sample was assayed in quadruplicate. The rate of change in absorbance recorded for the blank $(0.1 \%$ Triton X-100 in PBS) was subtracted from the sample rates to avoid interference from nonenzymatic conjugation. The results were expressed in $\mathrm{mM} / \mathrm{min} / \mathrm{mL}$ of sample.

\section{Tumor Necrosis Factor- $\alpha$ (TNF- $\alpha$ ) and Interleukin-6 (IL-6) Assessment}

TNF- $\alpha$ and IL- 6 concentrations were measured in $100 \mu \mathrm{L}$ previously diluted (1:5 with PBS buffer) blood plasma samples. For TNF- $\alpha$ measurements, TNF alpha Human Uncoated ELISA Kit with Plates was used (catalog number \#88-7346-76, ThermoFisher Scientific). For IL-6 measurements in plasma samples, IL-6 Human Uncoated ELISA Kit with Plates was used (catalog number \#887066-76, ThermoFisher Scientific). The results were expressed in $\mathrm{pg} / \mathrm{mL}$ of sample.

\section{Cholinesterase (ChE) Activity Assessment}

The following samples: whole blood, red blood cells, and plasma, were evaluated for ChE activity following Ellman's method. ${ }^{38}$ Briefly, the obtained samples of whole blood, erythrocyte suspensions, and plasma were diluted 1:300 in potassium phosphate buffer (P-P buffer) (100 mM, pH 8.0) with low detergent content $(0.01 \%$ Triton X-100). $100 \mu \mathrm{L}$ of the dilution was transferred into a 96-well microtiter plate with $100 \mu \mathrm{L}$ of $1 \mathrm{mM}$ substrate acetylthiocholine chloride in Ellman's reagent (5,5'-dithiobis-(2-nitrobenzoic acid) (DTNB) in $250 \mathrm{mM}$ P-P buffer, $\mathrm{pH}$ 7.4). Absorbance values were measured at $420 \mathrm{~nm}$ using a spectrophotometer (BioTek, Cytation 3, Bad Friedrichshall, Germany) for 30 cycles (at 2-minute intervals, for 60 minutes). All measurements were performed at room temperature in quadruplicate. The rate of change in absorbance recorded for blanks $(0.01 \%$ Triton $\mathrm{X}-100$ in $100 \mathrm{mM}$ P-P buffer) was subtracted from the sample rates to avoid interference from non-enzymatic reactions. The results were expressed in $\mathrm{mM} / \mathrm{min} / \mathrm{mL}$ of sample. 


\section{Assessment of the Standard Blood Hematological and Biochemical Parameters}

Laboratory blood sample analysis was performed for the following hematological parameters: blood cell counts for erythrocytes, platelets, leukocytes, MPV and the following biochemical parameters: lipidogram - cholesterol, HDLcholesterol, LDL-cholesterol, triglycerides and CRP. Laboratory blood sample analyses were performed at the Adria Lab d.o.o. Diagnostic Laboratory in Ljubljana, Slovenia. The concentrations of total cholesterol, HDLcholesterol, LDL-cholesterol, and triglycerides were measured by enzymatic colorimetric assays on a Roche/Hitachi Cobas ${ }^{\circledR} 6000$ system (Roche Diagnostic GmbH, Mannheim, Germany). MPV, erythrocyte and platelet counts were measured with flow cytometric light scattering and myeloperoxidase activity light scattering was used for measuring leukocytes count on Advia ${ }^{\circledR} 2120 \mathrm{i}$ Hematology System (Siemens Healthcare GmbH, Erlangen, Germany). Immunoturbidimetric method was used for CRP measurement on AU680 (Beckman Coulter, Inc., Brea, California).

\section{Statistical Analysis}

Data are presented as means (standard deviations) while differences are expressed as the percentage of decreased or increased values after physical effort in comparison to values before physical effort. Differences were compared by Student's $t$-test for dependent samples. The corresponding probability was calculated by using Excel v. 16.35 (Microsoft, Washington) software. Pearson's test was used for calculating the correlation coefficients between variables. The corresponding probability was calculated by using GraphPad Prism v. 8.4.1 (GraphPad Software, California). All tests were two-tailed, and values of $p<0.05$ were considered statistically significant.

\section{Results}

\section{Decrease of Measured Parameters After More Than 15 Hours of Recovery from Physical Effort}

On both sampling days (two days before marathon and $>15$ hours after marathon), CNV concentration in isolates was independent of gender and running distance (participants ran 10,21 or $42 \mathrm{~km}$ ). The effect of physical effort (longdistance running) on CNV concentration, GST activity in erythrocyte suspension and plasma samples, concentration of IL- 6 and TNF- $\alpha$ in plasma samples and ChE activity in erythrocyte suspension, whole blood and plasma samples, are shown in Figure 1. Results $>15$ hours after the marathon were compared with results two days before the marathon. We found a decrease in ChE activity in whole blood $(47 \%$; $\mathrm{p}<0.001)$, plasma $(34 \% ; \mathrm{p}<0.01)$ and erythrocyte suspension $(54 \% ; \mathrm{p}<0.001)$. We also found decrease in the concentration of CNVs in isolates $(46 \% ; \mathrm{p}<0.05)$ and in GST activity in erythrocyte suspension $(16 \% ; \mathrm{p}<0.01)$. There was no change in GST activity in plasma samples $(p=0.36)$. Plasma samples' concentration of inflammatory factor IL-6 statistically significantly decreased $>15$ hours after the marathon $(63 \% ; \mathrm{p}=0.04)$ but TNF- $\alpha$ did not $(\mathrm{p}=0.60)$.

\section{Correlation Between Measured Parameters After the Effort}

$\mathrm{CNV}$ concentration in isolates correlated with ChE activity in whole blood $(\mathrm{r}=0.821 ; \mathrm{p}<0.001)$, with $\mathrm{ChE}$ activity in erythrocyte suspension $(\mathrm{r}=0.820 ; \mathrm{p}<0.001)$ and with $\mathrm{ChE}$ activity in plasma $(\mathrm{r}=0.823 ; \mathrm{p}<0.001)$. Also, $\mathrm{CNV}$ concentration in isolates correlated with GST activity in erythrocyte suspension $(\mathrm{r}=0.824 ; \mathrm{p}<0.001)$. All measurements before and after the effort were taken into account in these correlations. We found positive correlation between CNVs concentration after the physical effort and MPV $(r=0.46 ; \mathrm{p}<0.05)$. CRP concentration after the running correlated with the running distance $(\mathrm{r}=0.56 ; \mathrm{p}<0.01)$. There were no correlations between $\mathrm{CNV}$ concentration in isolates and other hematological parameters (blood cell counts for erythrocyte suspensions, platelets, leukocytes and CRP concentration) and biochemical parameters (lipidogram - cholesterol, HDLcholesterol, LDL-cholesterol and triglycerides) (Table 1).

\section{Hematological and Biochemical Parameters}

More than 15 hours after physical effort the average values of lipidogram parameters (cholesterol, lipoproteins HDLcholesterol, LDL-cholesterol and triglycerides) and of blood cell counts (erythrocytes, platelets, and leukocytes) were all within the normal range for healthy individuals (Table 2). The average value of CRP was elevated; however, the increase was not statistically significant $(\mathrm{p}=0.050)$.

\section{Discussion}

With this study we provide experimental evidence that $>15$ hours after physical effort, the concentration of CNVs in 


\begin{tabular}{|c|c|c|c|c|c|c|c|c|c|c|c|}
\hline 选 & 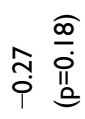 & 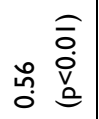 & 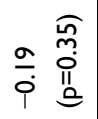 & 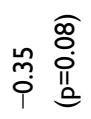 & 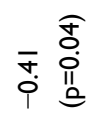 & م્ & 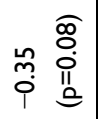 & 용 & ז̊ & o. & - \\
\hline 亥 & 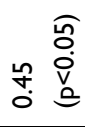 & 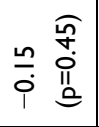 & 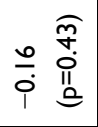 & 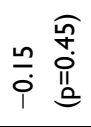 & 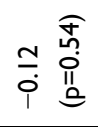 & 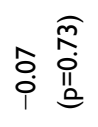 & 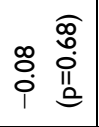 & $\stackrel{m}{m} \stackrel{0}{o}$ & 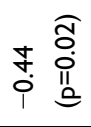 & - & \\
\hline 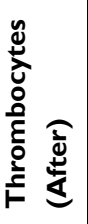 & 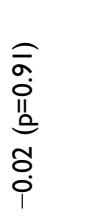 & 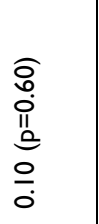 & 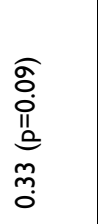 & $\begin{array}{l}\widehat{\overline{\bar{o}}} \\
\overline{11} \\
\stackrel{10}{0} \\
\stackrel{0}{0} \\
0\end{array}$ & 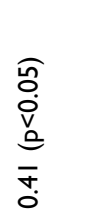 & 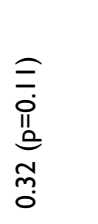 & $\begin{array}{l}\frac{a}{0} \\
\substack{0 \\
0} \\
\stackrel{m}{m} \\
0\end{array}$ & 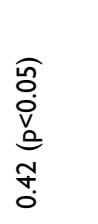 & - & & \\
\hline 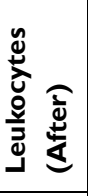 & 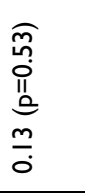 & 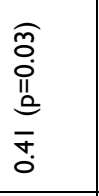 & 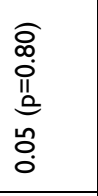 & 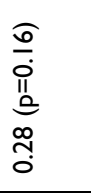 & $\begin{array}{l}0 \\
\stackrel{0}{0} \\
\text { II } \\
0 \\
0 \\
0 \\
0\end{array}$ & 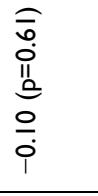 & $\begin{array}{l}\text { ह̂. } \\
0 \\
11 \\
0 \\
0 \\
0 \\
0 \\
0\end{array}$ & - & & & \\
\hline د & $\frac{\widehat{\tilde{p}}}{0} \stackrel{\substack{0 \\
0}}{\stackrel{0}{0}}$ & 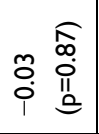 & 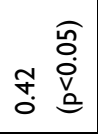 & 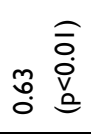 & 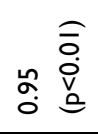 & 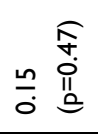 & - & & & & \\
\hline 古密 & 둥 & 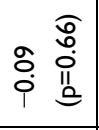 & 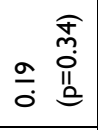 & 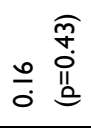 & 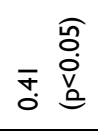 & - & & & & & \\
\hline 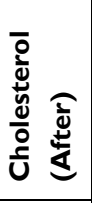 & $\begin{array}{l}\widehat{0} \\
0 \\
0 \\
0 \\
01 \\
0 \\
0 \\
0 \\
0 \\
0\end{array}$ & 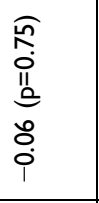 & 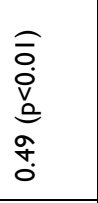 & $\begin{array}{l}\widehat{\bar{o}} \\
\dot{0} \\
\text { v } \\
\hat{0} \\
\hat{o} \\
0\end{array}$ & - & & & & & & \\
\hline 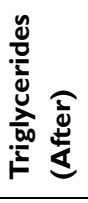 & 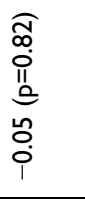 & $\begin{array}{l}\widehat{\sigma} \\
\text { o. } \\
\text { II } \\
0 \\
0 \\
0 \\
0\end{array}$ & 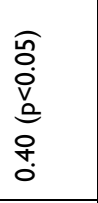 & - & & & & & & & \\
\hline 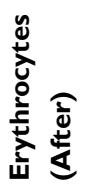 & 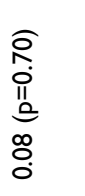 & 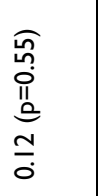 & - & & & & & & & & \\
\hline 离 & 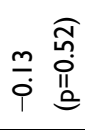 & - & & & & & & & & & \\
\hline 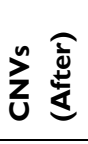 & - & & & & & & & & & & \\
\hline & 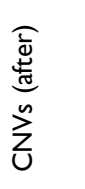 & 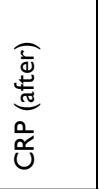 & 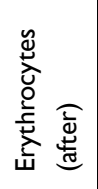 & 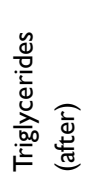 & 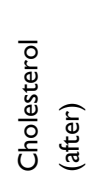 & 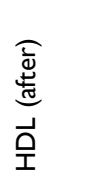 & 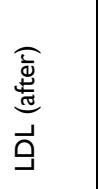 & 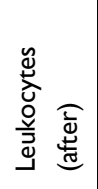 & 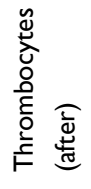 & 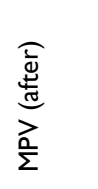 & 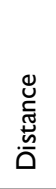 \\
\hline
\end{tabular}


Table 2 Average Values of Lipidogram Parameters and Blood Cell Counts in Blood Samples Taken More Than I5 Hours After Physical Effort

\begin{tabular}{|c|c|c|c|}
\hline Variables & Study Data & Reference Value & I-p value \\
\hline$N$ (number of samples) & 27 (M: I2, F: 15) & & \\
\hline CRP $[\mathrm{mg} / \mathrm{L}]$ & $7.3 \pm 5.9$ & $<5$ & 050 \\
\hline Cholesterol [mmol/L] & $5.1 \pm 1.0$ & $4.0-5.2$ & 0.526 \\
\hline HDL-cholesterol [mmol/L] & $\begin{array}{l}\text { M: } 1.5 \pm 0.2 \\
\text { F: } 1.8 \pm 0.3\end{array}$ & $\begin{array}{l}M>1.4 \\
F>1.6\end{array}$ & $\begin{array}{l}M: 0.653 \\
F:>0.990\end{array}$ \\
\hline LDL-cholesterol [mmol/L] & $3.1 \pm 0.8$ & $2.0-3.5$ & $>0.990$ \\
\hline Triglycerides [mmol/L] & $0.8 \pm 0.3$ & $0.6-1.7$ & $>0.990$ \\
\hline Erythrocytes $\left[10^{12} / \mathrm{L}\right]$ & $\begin{array}{l}\text { M: } 5.0 \pm 0.3 \\
\text { F: } 4.5 \pm 0.3\end{array}$ & $\begin{array}{l}\text { M: } 4.5-6.3 \\
F: 4.2-5.4\end{array}$ & $\begin{array}{l}M:>0.990 \\
F:>0.990\end{array}$ \\
\hline Platelets $\left[10^{9} / \mathrm{L}\right]$ & $249.2 \pm 44.7$ & $140-340$ & $>0.990$ \\
\hline Leukocytes $\left[10^{9} / \mathrm{L}\right]$ & $6.9 \pm 1.4$ & $4.0-10.0$ & $>0.990$ \\
\hline
\end{tabular}

Notes: Study data are presented by the average value \pm standard deviation. $\mathrm{p}$-values were calculated by the one-sample $t$-test.

Abbreviations: CRP, C reactive protein; HDL, high density lipoprotein; LDL, low density lipoprotein; $M$, male; F, female.

isolates from blood decreased concomitantly with activity of ChE and GST, as well as with inflammatory factor IL-6 concentration in blood samples (Figure 3).

Our results show that after $>15$ hours of recovery, concentration of CNVs decreased below the basal concentration. Most of the previous studies reported increase in the concentration of CNVs in blood isolates during or immediately after physical effort ${ }^{2,6,9,13,17,18}$ or return of the concentration of CNVs to baseline after some hours. ${ }^{9,13,17,18}$ Brahmer et al $(2019)^{2}$ observed 21 healthy male athletes subjected to an incremental cycling test until exhaustion; blood was drawn before, during, and immediately after the test. They found that the concentration of CNVs in isolates from blood increased during (with highest concentrations at peak effort) and immediately after cycling exercise. Frühbeis et al (2015) ${ }^{13}$ assessed CNVs released into the circulation of 12 healthy individuals who were cycling and running until exhaustion. The concentration of CNVs was on average $1.5(0.2)$ times higher immediately after cycling compared to the concentration of CNVs before cycling. However, already after $90 \mathrm{~min}$ of rest, concentration of CNVs returned to baseline values before cycling. Further, follow-up in two participants showed that the respective values measured 6 hours and one day after physical effort stayed at baseline. Wilhelm et al $(2016)^{17}$ collected blood samples from 9 healthy young men before and during heavy cycling exercise as well as one hour after the recovery period. They followed CNVs by marking the receptors characteristic for platelets and endothelial cells. They found more than two-fold $(\mathrm{p}<0.05)$ increase in concentration of CNVs carrying receptors characteristic for platelets and return to baseline after the one-hour recovery period, whereas concentration of CNVs carrying receptors characteristic for endothelial cells was on average unchanged. Chaar et al $(2011)^{6}$ noticed app. $6 \%(\mathrm{p}<0.05)$ increase in concentration of CNVs carrying receptors characteristic for platelets of seven individuals immediately after strenuous exercise which persisted after a two-hour recovery period. A study reported by Sossdorf et al $(2016)^{18}$ considered 16 trained and untrained individuals who performed 90 minutes of cycling at $80 \%$ of their individual anaerobic threshold. Blood samples were collected before, immediately after, 45 minutes after, and two hours after cycling. Before cycling no differences between trained and untrained individuals were observed in the concentration of CNVs. After exercise, the concentration of CNVs increased, being highest at 45 minutes and two hours in trained and untrained individuals, respectively. While in untrained subjects CNVs peaked after two hours of recovery, in trained subjects Augustine et al (2014) ${ }^{9}$ measured concentration of CNVs in blood samples of 107 individuals before, immediately after, and one hour after undergoing dobutamine stress echocardiogram. Their analysis has shown that in healthy participants, the concentration of procoagulant CNVs carrying receptors characteristic for erythrocytes, platelets and endothelial cells was found 


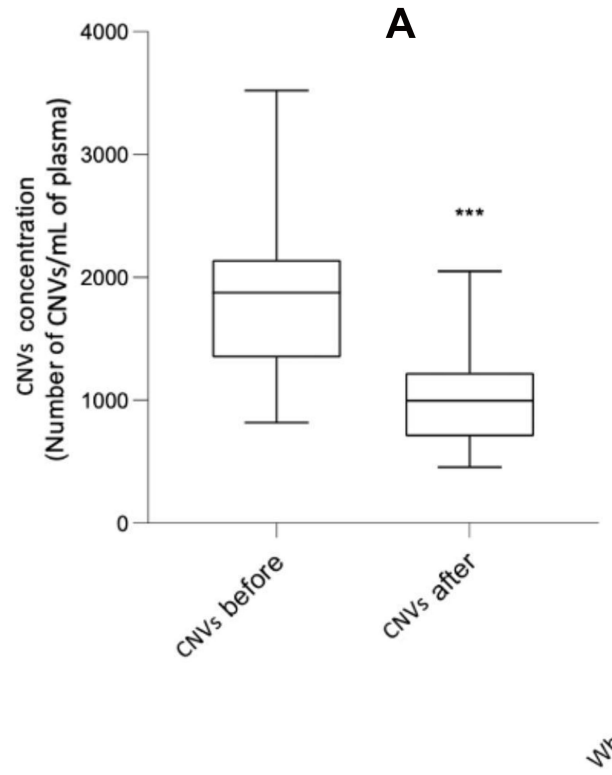

C

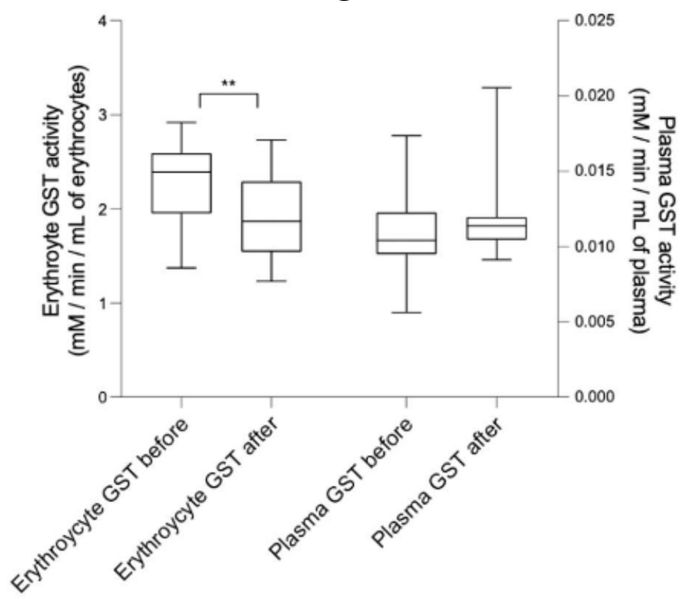

B

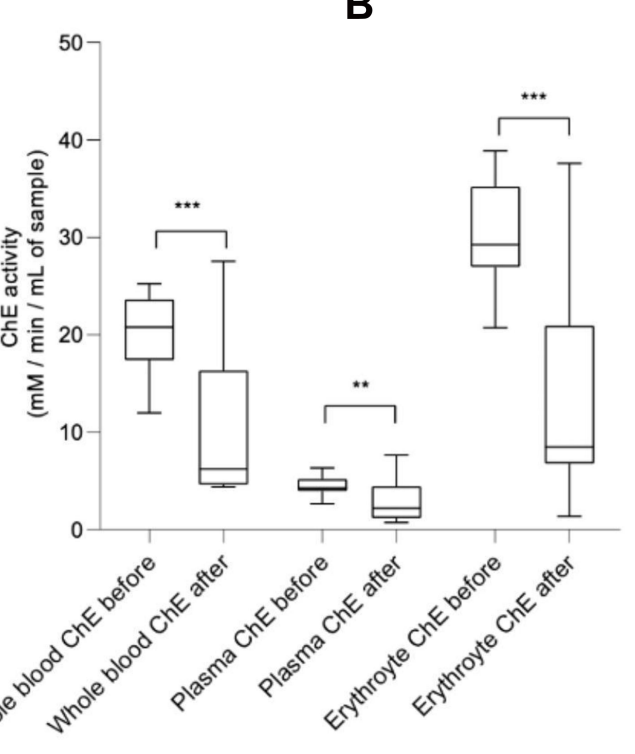

D

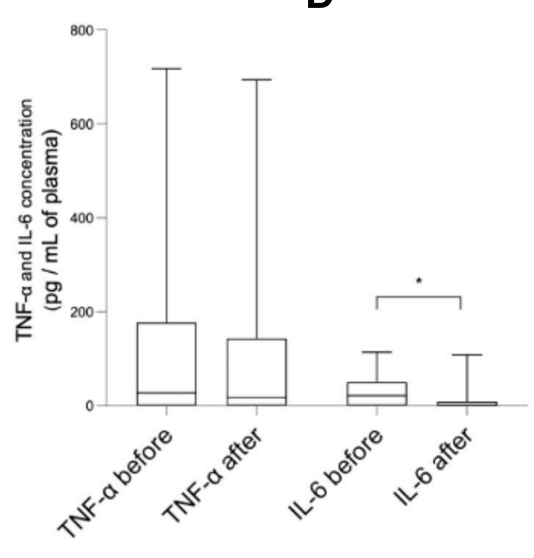

Figure 3 The effect of physical effort on concentration of microparticles and measured physiological state-related parameters in blood. (A) CNVs concentration in I mL plasma samples two days before and $>15$ hours after physical effort. (B) Activity of ChE $(\mathrm{n}=24)$ (in $\mathrm{mM} / \mathrm{min} / \mathrm{mL}$ of sample) two days before and $>15$ hours after physical effort in plasma, erythrocyte suspension and whole blood samples. (C) Activity of GST ( $\mathrm{n}=24$ ) (in $\mathrm{mM} / \mathrm{min} / \mathrm{mL}$ of sample) in plasma and erythrocyte suspension samples two days before and $>15$ hours after physical effort. Scale on the left represents values of plasma GST activity and scale on the right represents values of erythrocyte suspension GST activity. (D) Concentration of IL-6 and TNF- $\alpha(n=26)$ (in $\mathrm{pg} / \mathrm{mL}$ of plasma) two days before and $>15$ hours after physical effort. Statistical differences before and after physical effort at: $* p<0.05$, ** $p<0.01$ and $* * * p<0.001$.

Abbreviations: CNVs, cellular nanovesicles; ChE, cholinesterase; GST, Glutathione S-transferase; TNF- $\alpha$, tumor necrosis factor; IL-6 -interleukin 6.

elevated immediately after cardiac stress. After one hour of rest, procoagulant and platelet CNVs returned to baseline, erythrocyte-derived CNVs started to decrease and endothelial cell-derived CNVs fell below the baseline. Except Augustine et al (2014), ${ }^{9}$ who reported a decreased concentration of CNVs one hour after rest, most of the previous studies described return of CNVs concentration to baseline after rest. ${ }^{2,6,13,17,18}$ However, the concentration of CNVs was measured immediately after the physical effort or after a shorter recovery period. In two individuals only, ${ }^{13}$ the observation time was comparable to ours: in these two participants, the concentration of CNVs measured one day after the effort was found to be at baseline. Concentration of CNVs depends on the duration of recovery period. However, our study reports CNVs decrease after $>15$ hours recovery period, below the baseline. This indicates a beneficial effect of long-distance running exercise. CNV concentration $>15$ hours after rest decreased concomitantly with other measured state-related parameters: erythrocyte suspension GST activity, whole blood, plasma and erythrocyte suspension ChE activity and IL-6 plasma concentration. This indicates that $\mathrm{CNV}$ concentration measurements 
give us information about the response of the human body to physiological stress and inflammatory process after physical effort.

It was suggested that EVs present in the circulation might help cells to maintain hemostasis with assistance of disposal of cellular waste products generated as a result of physical effort; EVs, especially exosomes, released into circulation, could have a role in regulating physiological adaptation processes in response to physical activity and therefore have beneficial health-protective effect. ${ }^{13}$ On the other hand, increased concentration of CNVs in isolates from blood was connected with increased risk for blood clot formation in blood vessels and therefrom deriving increased risk for thromboembolic events ${ }^{39}$ and also with increased risk for other cardio-metabolic diseases, such as Type 2 diabetes mellitus. ${ }^{40}$ Monitoring concentration of $\mathrm{CNVs}$ is clinically relevant as it is indicated that it may be informative in assessment of instantaneous risk for thromboembolic events ${ }^{39,40}$ and glucose tolerance. ${ }^{41}$ Further studies are needed to improve CNV isolation and assessment methods and to link the measurements of CNVs in isolates from blood to various pathologies. ${ }^{40}$

Erythrocyte suspension GST activity dropped below the initial activity after $>15$ hours of rest. Erythrocyte suspension GST has been proposed as a potential marker for oxidative stress ${ }^{25}$ and CNVs formation is considered to be provoked by oxidative stress. ${ }^{15}$ Similarly, Yarana et al $(2017)^{42}$ suggested that during oxidative stress, oxidized proteins are formed, and cells release EVs as a compensatory mechanism to remove them and to maintain homeostasis. Connection between GST activity in erythrocyte suspension and concentration of CNVs in blood isolates can indicate a decrease in oxidative stress $>15$ hours after recovery period. Evelo et al $(1992)^{4}$ documented changes in erythrocyte suspension GST activity in 41 individuals who trained for 40 weeks to run a half marathon race. They reported that the activity of erythrocyte suspension GST decreased one day after the marathon and was restored four days later. Our results are consistent with Evelo et al's (1992), ${ }^{4}$ indicating absence of oxidative stress after $>15$ hours of rest.

After $>15$ hours of rest, ChE activity in blood, plasma and erythrocyte suspension dropped below initial values, concomitantly with decrease of CNVs concentration. Connection between AChE activity in erythrocyte suspension and concentration of $\mathrm{CNV}$ was previously considered in other studies, ${ }^{42-46}$ in which AChE activity was used as an exosome marker. Release of small vesicles into extracellular medium during maturation of reticulocytes into erythrocytes, was monitored. ${ }^{46}$ Reticulocytes get rid of membrane proteins that they no longer need with release of small vesicles - exosomes, enriched with $\mathrm{AChE}$ and this phenomenon contributes to decrease of $\mathrm{AChE}$ from the erythrocyte surface. ${ }^{46}$ Liao et al $(2019)^{47}$ suggested that $\mathrm{ChE}$ activity can be used as a marker for erythrocyte derived CNVs. Duchnowicz et al (2018) ${ }^{23}$ suggested that decrease in erythrocyte suspension $\mathrm{AChE}$ activity may reflect an increased response of the human body to oxidative stress, which was also confirmed in our study with drop of $\mathrm{ChE}$ and GST activity in erythrocyte suspension after $>15$ hours of rest. Considering 15 individuals, Zimmer et al (2011) ${ }^{22}$ found an increase of BuChE activity in plasma and $\mathrm{AChE}$ activity in erythrocyte suspension. They concluded that a single session of physical exercise performed on a treadmill at a speed of $7 \mathrm{~km} / \mathrm{h}$ for 30 minutes was sufficient to stimulate the release of $\mathrm{BuChE}$ in the plasma. However, they did not observe changes in the BuChE activity after recovery period. Chamera et al $(2015)^{3}$ reported that $\mathrm{ChE}$ activity in blood samples of 8 female football players 15 minutes after a one-hour run was higher than its baseline values, but lower than ChE activity immediately after the run. The results obtained by Chamera et al $(2015)^{3}$ indicate that $\mathrm{ChE}$ activity starts decreasing during the recovery period, and our results also indicate a decrease in ChE activity after $>15$ hours of rest. Also, our results suggest that $\mathrm{ChE}$ is a potential indicator of response of the human body to inflammation after physical effort, since ChE activity was lower after $>15$ hours of rest concomitantly with CNVs concentration, erythrocyte suspension GST activity, and IL-6 concentration.

We found a decrease in IL-6 concentration in blood plasma samples after $>15$ hours of rest. Chaar et al (2011) previously reported the impact of inflammatory processes on vesiculation. Other authors found a relationship between running intensity, duration and level of fitness and elevated plasma IL-6 concentration. ${ }^{20}$ It was also reported $^{5,6,47}$ that immediately after physical effort, plasma IL-6 concentration increased. Chaar et al $(2011)^{6}$ and Fischer et al (2004) ${ }^{44}$ reported that IL-6 plasma concentration began to lower after two hours of rest but remained elevated compared to basal values. Fischer et al (2004) ${ }^{48}$ also monitored IL-6 plasma concentration after 20 hours of rest and reported that it almost reached basal concentration. Mendham et al $(2010)^{5}$ reported that after one day of recovery, plasma IL-6 concentration decreased slightly below basal values, which is in agreement with our results. 
Decrease in plasma IL-6 concentration indicates beneficial effects of physical effort on the human body after $>15$ hours of rest. Individuals who are regularly physically active have lower basic concentrations of IL- $6^{49}$ and therefore have lower risk of diseases associated with low-grade inflammation: cardiovascular disease ${ }^{19}$ and Type 2 diabetes mellitus. ${ }^{20}$

Plasma TNF- $\alpha$ concentration was not changed after $>15$ hours of rest and that is similar to previously reported results for TNF- $\alpha .{ }^{50}$ TNF- $\alpha$ in general does not increase with exercise. ${ }^{20}$ Timmerman et al $(2008)^{51}$ assessed blood samples of 15 physically active individuals at baseline and after 12 weeks of regular training. They reported 13\% decrease in plasma TNF- $\alpha$ concentration, indicating that regular physical effort decreases concentration of TNF- $\alpha$. TNF- $\alpha$ is mainly produced and works locally and is not always reflected in its systemic levels. However, chronically elevated IL-6 and CRP concentrations are likely to reflect local ongoing TNF- $\alpha$ production and from this aspect, TNF- $\alpha$ is also connected with systemic low-grade inflammation. ${ }^{20}$

After the marathon, CRP concentrations were found higher than proposed criteria for healthy individuals (Table 2). However, elevated CRP concentrations were not statistically significant. Furthermore, CRP concentrations were positively correlated to the running distance (Table 1). Other authors reported increase in CRP concentrations after longer period of rest: Mendham et al (2010) and Fischer et al (2004) ${ }^{48}$ reported increase in CRP concentrations one day and 20 hours after physical effort. Regular physical activity is reported to reduce CRP concentrations and therefore may suppress systemic low-grade inflammation. ${ }^{20}$

We found correlation between the concentration of CNVs in isolates and MPV (Table 1), indicating that in healthy physically active individuals, platelets are the major source of CNVs in blood isolates. ${ }^{17}$ Namely, MVP is considered to be a marker of platelet activity which may lead to formation of CNVs in blood isolates. ${ }^{32}$

We used the most prevalent technique for CNVs detection and counting, flow cytometry. Since measurements with a flow cytometer are restricted to CNVs above the size of $500 \mathrm{~nm}$, it is unclear to what extent $<500 \mathrm{~nm}$ CNVs contributed to vesicle enumeration in our study. ${ }^{51}$

There are some known biases in the isolation of plasma CNVs samples, therefore it must be taken into consideration how the person should prepare for CNVs sampling. ${ }^{29}$ Present instructions include overnight fasting, ${ }^{34,52}$ since it has been previously shown that the concentration of CNVs in peripheral blood correlates with lipoproteins, including high density lipoprotein (HDL), low density lipoprotein (LDH), triglycerides (TGL) ${ }^{2}$ and exclusion of activities that have already been found to interfere with the results (eg, physical effort, travel with airplane, long distance driving, irradiation). To avoid additional formation of CNVs, donors were asked to use car or public transport to the blood sampling site.

In our study, measured lipoproteins (triglycerides, total cholesterol, HDL-cholesterol and LDL-cholesterol) were within the normal range after physical effort and the fasting state of the participants contributed to the reduction of error due to lipoprotein occurrence in the samples. Other parameters that can interfere with measuring the concentration of CNVs are blood cell count of erythrocytes, platelets and leukocytes. ${ }^{2}$ In our study cell count of blood cells was within the normal range and also, there was no statistically significant correlation between blood cell count and concentration of CNVs after physical effort. It can be expected that the in vivo release of CNVs also depends on the physiological background of the individuals and may be influenced by parameters like age, immune status, hormone levels or metabolic state. ${ }^{2}$ It should be kept in mind that the isolation procedures themselves affect the CNVs' formation and that the indirect effects of the media in which the CNVs are processed are also a contributing factor. ${ }^{34}$ Participants in our study had not eaten until after blood collection app 20 hours after the marathon. Therefore, their bodies could not regenerate in the first hours after physical effort as it would have if they had eaten usual amount of food. Improved methods for CNVs isolation and assessment and improved knowledge on specific role in tissue crosstalk and action mechanisms in target cells, may help with future diagnostic and therapeutic applications of CNVs. Also, a better understanding CNVs' cellular sources, targets and signaling components, can help to master healthpromoting effects associated with physical activity.

In statistical analysis we considered that the measured values of parameters were normally distributed, however, a more rigorous analysis showed that the data for two parameters that showed statistically significant difference by assuming normality (IL-6 and $\mathrm{ChE}$ in whole blood) could not be transformed to normality. The Wilcoxon Sign Rank test of the transformed data yielded the p-values for these two parameters as 0.073 and $<0.001$, respectively. However, as at least three independently 
assessed parameters (CNVs, GST in erythrocyte suspension and $\mathrm{ChE}$ in plasma and in erythrocyte suspension) complied to rigorous statistical analysis and showed a decrease after the effort, this does not affect the main conclusion of our study - a decrease in the concentration of CNVs in isolates $>15$ hours after physical effort.

In interpreting the results, it should be borne in mind that athletes had been training and preparing for the marathon, which affected the results also before the effort. Furthermore, the population analyzed was heterogeneous with respect to age and sex. However, it should be acknowledged that participation in such a study requires considerable input of the participants and we considered ourselves fortunate that 27 athletes consented to participate. Fasting for two days before the marathon may not have been optimal for their achievement at the marathon and it was particularly difficult for the participants to fast 15 hours on the night after the marathon until the second sampling was performed the next morning, especially for those who had run $42 \mathrm{~km}$. Fasting is however necessary for accurate assessment of CNV concentration since the lipid droplets after food intake present artefacts in determination of the concentration of CNVs. Nevertheless, we were able to show the effect of running also on this heterogeneous population. It is expected that even more information could be obtained by studying homogeneous groups and it seems likely that the differences also would have been obtained in plasma GST and TNF- $\alpha$ (Figure 3C and D) in groups matching in sex, age, and physical effort.

\section{Conclusion}

We obtained lower concentration of CNVs in isolates from peripheral blood $>15$ hours after physical effort with lower values of other measured physiological state-related parameters: whole blood, plasma and erythrocyte suspension ChE activity, erythrocyte suspension GST activity and IL-6 plasma concentrations. This suggests that CNVs concentration and $\mathrm{ChE}$ activity have the potential to indicate impact of physical effort on inflammation in the human body. Also, concentration of the measured parameters depends on the duration of recovery period. According to previous reports, physical effort causes an increase in concentration of CNVs, but according to our results, the concentration of CNVs in isolates decreases after rest and may reach values lower than initial ones. With this, our study provides novel insights into the beneficial effects of physical effort on the human body and guidance about physical effort for donors before blood collection in procedures focusing on CNV-containing diagnostic and therapeutic compounds.

\section{Acknowledgments}

Results of the study were presented clearly, honestly, and without fabrication, falsification, or inappropriate data manipulation. Results of the present study do not constitute endorsement by The American College of Sports Medicine (ACSM). Authors acknowledge support from EU Commission (Ves4us) and Slovenian Research Agency (P3-0388, P2-9232, P1-0132, J1-9162, P5-7098).

\section{Disclosure}

No conflicts of interest, financial or otherwise, are declared by the authors.

\section{References}

1. Whitham M, Parker BL, Friedrichsen M, et al. Extracellular vesicles provide a means for tissue crosstalk during exercise. Cell Metab. 2018;27(1):237-251.e4. doi:10.1016/j.cmet.2017.12.001

2. Brahmer A, Neuberger E, Esch-Heisser L, et al. Platelets, endothelial cells and leukocytes contribute to the exercise-triggered release of extracellular vesicles into the circulation. J Extracell Vesicles. 2019;8 (1):1615820. doi:10.1080/20013078.2019.1615820

3. Chamera T, Spieszny M, Klocek T, et al. Post-effort changes in activity of traditional diagnostic enzymatic markers in football players' blood. J Med Biochem. 2015;34(2):179-190. doi:10.2478/ jomb-2014-0035

4. Evelo CT, Palmen NG, Artur Y, Janssen GM. Changes in blood glutathione concentrations, and in erythrocyte glutathione reductase and glutathione S-transferase activity after running training and after participation in contests. Eur J Appl Physiol Occup Physiol. 1992;64 (4):354-358. doi:10.1007/bf00636224

5. Mendham AE, Donges CE, Liberts EA, Duffield R. Effects of mode and intensity on the acute exercise-induced IL-6 and CRP responses in a sedentary, overweight population. Eur J Appl Physiol. 2011;111 (6):1035-1045. doi:10.1007/s00421-010-1724-z

6. Chaar V, Romana M, Tripette J, et al. Effect of strenuous physical exercise on circulating cell-derived microparticles. Clin Hemorheol Microcirc. 2011;47(1):15-25. doi:10.3233/CH-2010-1361

7. Simioni C, Zauli G, Martelli AM, et al. Oxidative stress: role of physical exercise and antioxidant nutraceuticals in adulthood and aging. Oncotarget. 2018;9(24):17181-17198. doi:10.18632/oncotarget.24729

8. Witwer KW, Théry C. Extracellular vesicles or exosomes? On primacy, precision, and popularity influencing a choice of nomenclature. J Extracell Vesicles. 2019;8(1):1648167. doi:10.1080/20013078.2019.1648167

9. Augustine D, Ayers LV, Lima E, et al. Dynamic release and clearance of circulating microparticles during cardiac stress. Circ Res. Jan 2014;114(1):109-13. doi:10.1161/CIRCRESAHA.114.301904

10 Hurley JH, Boura E, Carlson LA, Różycki B. Membrane budding. Cell. Dec 2010;143(6):875-87. doi:10.1016/j. cell.2010.11.030

11. Sorkin R, Huisjes R, Bošković F, et al. Nanomechanics of extracellular vesicles reveals vesiculation pathways. Small. 2018;14(39):50. doi: 50

12. Oszvald Á, Szvicsek Z, Sándor GO, et al. Extracellular vesicles transmit epithelial growth factor activity in the intestinal stem cell niche. Stem Cells. 2020;38(2):291-300. doi:10.1002/stem.3113 
13. Frühbeis C, Helmig S, Tug S, Simon P, Krämer-Albers EM. Physical exercise induces rapid release of small extracellular vesicles into the circulation. J Extracell Vesicles. 2015;4(1):28239. doi:10.3402/jev. v4.28239

14. Morshed A, Karawdeniya BI, Bandara YMND, Kim MJ, Dutta P. Mechanical characterization of vesicles and cells: a review. Electrophoresis. 2020;41(7-8):449-470. doi:10.1002/elps.201900362

15. Borras C, Mas-Bargues C, Sanz-Ros J, et al. Extracellular vesicles and redox modulation in aging. Free Radic Biol Med. 2020;149:44-50. doi:10.1016/j.freeradbiomed.2019.11.032

16. Rakobowchuk M, Ritter O, Wilhelm EN, et al. Divergent endothelial function but similar platelet microvesicle responses following eccentric and concentric cycling at a similar aerobic power output. J Appl Physiol. 2017;122(4):1031-1039. doi:10.1152/japplphysiol.00602.2016

17. Wilhelm EN, González-Alonso J, Parris C, Rakobowchuk M. Exercise intensity modulates the appearance of circulating microvesicles with proangiogenic potential upon endothelial cells. Am $J$ Physiol Heart Circ Physiol. 2016;311(5):H1297-H1310. doi:10.1152/ajpheart.00516.2016

18. Sossdorf M, Otto GP, Claus RA, Gabriel HH, Lösche W. Cellderived microparticles promote coagulation after moderate exercise. Med Sci Sports Exerc. 2011;43(7):1169-1176. doi:10.1249/MSS.0b013e3182068645

19. Liem RI, Onyejekwe K, Olszewski M, et al. The acute phase inflammatory response to maximal exercise testing in children and young adults with sickle cell anaemia. $\mathrm{Br} J$ Haematol. 2015;171 (5):854-861. doi:10.1111/bjh.13782

20. Petersen AMW, Pedersen BK. The anti-inflammatory effect of exercise. J Appl Physiol. 2005;98(4):1154-1162. doi:10.1152/ japplphysiol.00164.2004

21. Villeda-González JD, Gómez-Olivares JL, Baiza-Gutman LA, et al. Nicotinamide reduces inflammation and oxidative stress via the cholinergic system in fructose-induced metabolic syndrome in rats. Life Sci. 2020;50:117585. doi:10.1016/j.1fs.2020.117585

22. Zimmer KR, Lencina CL, Zimmer AR, Thiesen FV. Influence of physical exercise and gender on acetylcholinesterase and butyrylcholinesterase activity in human blood samples. Int $J$ Environ Health Res. 2012;22(3):279-286. doi:10.1080/09603123.2011.634389

23. Duchnowicz P, Ziobro A, Rapacka E, Koter-Michalak M, Bukowska B. Changes in cholinesterase activity in blood of adolescent with metabolic syndrome after supplementation with extract from. Biomed Res Int. 2018;2018:5670145. doi:10.1155/2018/ 5670145

24. Mergani A, Mansour AA, Askar T, et al. Glutathione S-transferase pi-ile 105 val polymorphism and susceptibility to $\mathrm{t} 2 \mathrm{dm}$ in population from Turabah region of Saudi Arabia. Biochem Genet. 2016;54 (4):544-551. doi:10.1007/s10528-016-9740-2

25. Neefjes VM, Evelo CT, Baars LG, Blanco CE. Erythrocyte glutathione $\mathrm{S}$ transferase as a marker of oxidative stress at birth. Arch Dis Child Fetal Neonatal Ed. 1999;81(2):F130-3. doi:10.1136/ fn.81.2.f130

26. Jain S, Gautam V, Naseem S. Acute-phase proteins: as diagnostic tool. J Pharm Bioallied Sci. 2011;3(1):118-127. doi:10.4103/09757406.76489

27. Bradley JR. TNF-mediated inflammatory disease. J Pathol. 2008;214 (2):149-160. doi:10.1002/path.2287

28. Shephard RJ. Cytokine responses to physical activity, with particular reference to IL-6: sources, actions, and clinical implications. Crit Rev Immunol. 2002;22(3):165-182.

29. Venturella M, Carpi FM, Zocco D. Standardization of blood collection and processing for the diagnostic use of extracellular vesicles. Curr Pathobiol Rep. 2019;7(1):1-8. doi:10.1007/s40139-019-00189-3

30. Eichner NZM, Gilbertson NM, Musante L, et al. An oral glucose load decreases postprandial extracellular vesicles in obese adults with and without prediabetes. Nutrients. 2019;11(3):580. doi:10.3390/ nu11030580
31. Korniluk A, Koper-Lenkiewicz OM, Kamińska J, Kemona H, Dymicka-Piekarska V. Mean platelet volume (MPV): new perspectives for an old marker in the course and prognosis of inflammatory conditions. Mediators Inflamm. 2019;2019:9213074. doi:10.1155/ 2019/9213074

32. Lood C, Tydén H, Gullstrand B, et al. Decreased platelet size is associated with platelet activation and anti-phospholipid syndrome in systemic lupus erythematosus. Rheumatology (Oxford). 2017;56 (3):408-416. doi:10.1093/rheumatology/kew437

33. Štukelj R, Schara K, Bedina-Zavec A, et al. Effect of shear stress in the flow through the sampling needle on concentration of nanovesicles isolated from blood. Eur J Pharm Sci. 2017;98:17-29. doi:10.1016/j.ejps.2016.10.007

34. Šuštar V, Bedina-Zavec A, Stukelj R, et al. Nanoparticles isolated from blood: a reflection of vesiculability of blood cells during the isolation process. Int $J$ Nanomedicine. 2011;6:2737-2748. doi:10.2147/IJN.S24537

35. Šuštar V, Bedina-Zavec A, Stukelj R, et al. Post-prandial rise of microvesicles in peripheral blood of healthy human donors. Lipids Health Dis. 2011;10(1):47. doi:10.1186/1476-511X-10-47

36. Božič D, Sitar S, Junkar I, et al. Viscosity of plasma as a key factor in assessment of extracellular vesicles by light scattering. Cells. 2019;8 (9):1046. doi:10.3390/cells8091046

37. Mannervik B. The isoenzymes of glutathione transferase. $A d v$ Enzymol Relat Areas Mol Biol. 1985;57:357-417. doi:10.1002/ 9780470123034.ch5

38. Ellman GL, Courtney KD, Andres V, Feather-Stone RM. A new and rapid colorimetric determination of acetylcholinesterase activity. Biochem Pharmacol. 1961;7(2):88-95. doi:10.1016/0006-2952(61) 90145-9

39. Weber A, Liu SS, Cardone L, et al. The course of circulating small extracellular vesicles in patients undergoing surgical aortic valve replacement. Biomed Res Int. 2020;2020:6381396. doi:10.1155/ 2020/6381396

40. Vijay A, Garg I, Ashraf MZ. Perspective: DNA copy number variations in cardiovascular diseases. Epigenet Insights. 2018;11:2516865718818839. doi:10.1177/2516865718818839

41. Giannella A, Radu CM, Franco L, et al. Circulating levels and characterization of microparticles in patients with different degrees of glucose tolerance. Cardiovasc Diabetol. 2017;16(1):118. doi:10.1186/s12933-017-0600-0

42. Yarana C, St Clair DK. Chemotherapy-induced tissue injury: an insight into the role of extracellular vesicles-mediated oxidative stress responses. Antioxidants. 2017;6(4). doi:10.3390/antiox6040075

43. Cantin R, Diou J, Bélanger D, Tremblay AM, Gilbert C. Discrimination between exosomes and HIV-1: purification of both vesicles from cell-free supernatants. J Immunol Methods. 2008;338 (1-2):21-30. doi:10.1016/j.jim.2008.07.007

44. Johnstone RM, Adam M, Hammond JR, Orr L, Turbide C. Vesicle formation during reticulocyte maturation. Association of plasma membrane activities with released vesicles (exosomes). $J$ Biol Chem. 1987;262(19):9412-9420.

45. Park IW, He JJ. HIV-1 is budded from CD4+ T lymphocytes independently of exosomes. Virol J. 2010;7(1):234. doi:10.1186/1743422X-7-234

46. Rieu S, Géminard C, Rabesandratana H, Sainte-Marie J, Vidal M. Exosomes released during reticulocyte maturation bind to fibronectin via integrin alpha4beta1. Eur J Biochem. 2000;267(2):583-590. doi:10.1046/j.1432-1327.2000.01036.x

47. Liao Z, Jaular LM, Soueidi E, et al. Acetylcholinesterase is not a generic marker of extracellular vesicles. J Extracell Vesicles. 2019;8(1):1628592. doi:10.1080/20013078.2019.1628592

48. Fischer CP, Hiscock NJ, Penkowa M, et al. Supplementation with vitamins $\mathrm{C}$ and $\mathrm{E}$ inhibits the release of interleukin- 6 from contracting human skeletal muscle. J Physiol. 2004;558(Pt 2):633-645. doi:10.1113/jphysiol.2004.066779 
49. Maggio M, Guralnik JM, Longo DL, Ferrucci L. Interleukin-6 in aging and chronic disease: a magnificent pathway. J Gerontol a Biol Sci Med Sci. 2006;61(6):575-584. doi:10.1093/gerona/61.6.575

50. Timmerman KL, Flynn MG, Coen PM, Markofski MM, Pence BD. Exercise training-induced lowering of inflammatory (CD14+CD16+) monocytes: a role in the anti-inflammatory influence of exercise? J Leukoc Biol. 2008;84(5):1271-1278. doi:10.1189/jlb.0408244
51. Erdbrügger U, Lannigan J. Analytical challenges of extracellular vesicle detection: a comparison of different techniques. Cytometry A. 2016;89(2):123-134. doi:10.1002/cyto.a.22795

52. Eichner NZM, Erdbrügger U, Malin SK. Extracellular vesicles: a novel target for exercise-mediated reductions in type 2 diabetes and cardiovascular disease risk. $J$ Diabetes Res. 2018;2018:7807245. doi: $10.1155 / 2018 / 7807245$

\section{Publish your work in this journal}

The International Journal of Nanomedicine is an international, peerreviewed journal focusing on the application of nanotechnology in diagnostics, therapeutics, and drug delivery systems throughout the biomedical field. This journal is indexed on PubMed Central, MedLine, CAS, SciSearch ${ }^{\mathbb{R}}$, Current Contents ${ }^{\mathbb{R}} /$ Clinical Medicine, $^{2}$
Journal Citation Reports/Science Edition, EMBase, Scopus and the Elsevier Bibliographic databases. The manuscript management system is completely online and includes a very quick and fair peer-review system, which is all easy to use. Visit http://www.dovepress.com/ testimonials.php to read real quotes from published authors. 